\title{
PERFORMANCE ANALYSIS OF MANET ROUTING PROTOCOLS
}

\author{
${ }^{1}$ Irfan Ahmad Rasooly, ${ }^{2}$ Fahad Masood, ${ }^{3}$ Arbab Wajid Ullah Khan, ${ }^{4}$ Riaz Ahmad Ziar, ${ }^{5}$ Mohammad Saber Niazy \\ ${ }^{1,5}$ Dept. of Computing and Technology, Khurasan University, Nangarhar, Afghanistan \\ ${ }^{2,3}$ Dept. of Computing and Technology, Abasyn University, Peshawar Pakistan \\ ${ }^{4}$ Faculty of Engineering and Technology, Kardan University, Kabul, Afghanistan
}

\begin{abstract}
Mobile Ad-hoc networks are self- reliant system of mobile phones associated by mobile ad-hoc links. All hops in the framework collaborate so as to effectively route packets in multi-hop networks. Because of the eccentric versatility of hops, the system topology may change continually. Ad-hoc systems are fabricated and utilized as convenient in incredibly progressive situations, not really with the assistance of an earlier existing structure. Due to the none-centralized system routing is the essential issue for MANET. Different routing protocols have been developed in recent decade like AODV, DYMO, DSDV and DSR etc. This research study shows the simulation analysis of reactive, proactive and hybrid routing protocols. The performance matrices were analyzed packet ratio delivery (PDR), throughput, end - to - end delay and Normalized routing load (NRL). NS -

2.35 network simulation has been used for overall study experiments with respect to discrete number of hops and different pause time. From the experiment it is come to terms on that DSDV and DSR are performs well in all performance matrices.
\end{abstract}

Keywords: $A O D V, D S D V, D S R, N R L$, Pause time.

\section{INTRODUCTION}

Mobile ad-hoc network is a self-creating network, in which structure is automotive. These hops are undertaken to comply with the typical bump of radio communication channels, multi hops support, multi way blurring and so forth. MANET is a set of remote mobile hops that progressively makes the system without fixed physical foundation and incorporated access point. The mobile hops that are in radio scope of one another can straightforwardly convey, though others needs the guide of middle of the road hops to route their packet. The usefulness of MANET is learns the routes and retains up the routs with the cooperation of routers. In such networks hops are fit for moving and planning with their neighbors. Routing in MANETs is a one of the progressively and requesting task and has gotten a lot of mindfulness from researchers around the worldwide. To conquer this issue, a different number of routing classes have been presented and the number is as yet rising step by step fastly in [2017].

Section II presents the related work. Section III defines the simulations setup. Section IV presents the results and discussions and the conclusion of this research will be explained in section $\mathrm{V}$.

\subsection{WIRELESS NETWORKS}

Remote systems permit adaptable association between applicants who are in different spots. Besides, the system can be extended anyplace or working with short of a wired association between the hops. Wireless systems are part into two types: fixed infrastructure systems and wireless systems (less infrastructure networks) in [2015].

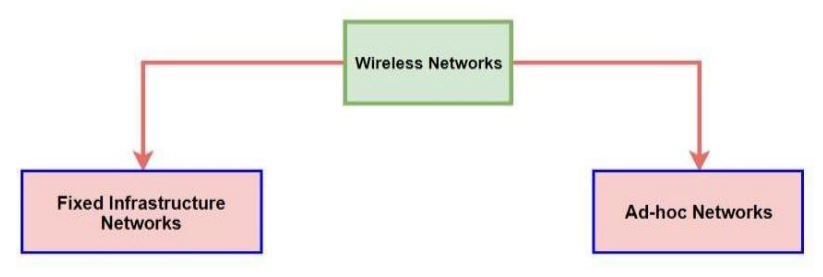

Figure 1. Wireless Networks

a) Fixed Infrastructure Network: Infrastructure manner is an 802.11 systems administration structure in which gadgets communicates with one another with the help third party devices. In Infrastructure mode, remote gadgets can communicate with one another or can transfer with a wired system. When one main device is associated with wired system and a lot of wireless stations it is alluded to as a basic service set (BSS). Most corporate wireless LANs work in fixed mode since they expect access to the wired LAN so as to utilize services like e.g. file server or printers.

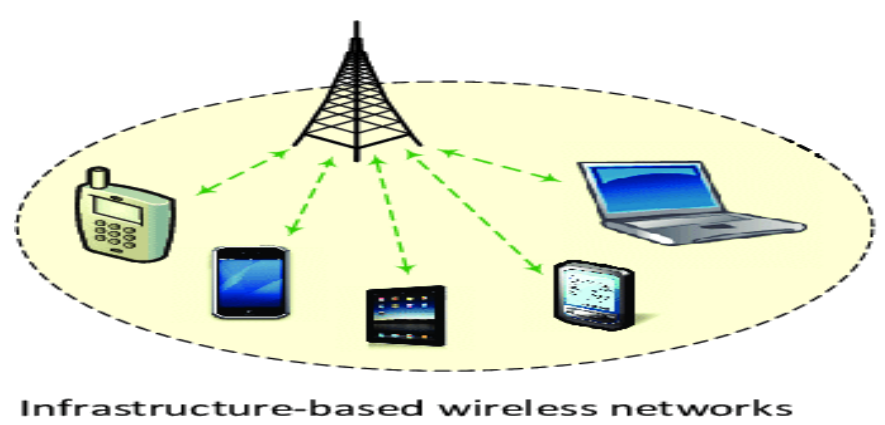

Figure 2. Fixed Infrastructure Network

b) Wireless Network: This type of systems is comprehended as mobile wireless network (MANET) which can make connections with one another without the help from main devices. That is the MANET which is a none- 
centralized kind of wireless network. At another way, the system is Ad hoc since it doesn't rely upon a fixed structure.

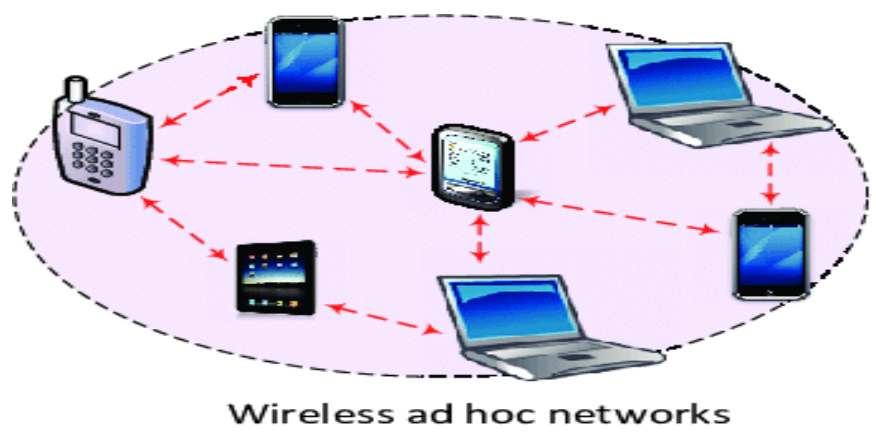

Figure 3. Ad-hoc Networks

\subsection{CHARACTERISTICS OF MANETS MANET}

has a few qualities that build it promoted wherever without fail. These qualities that are attractive in ad-hoc routing protocols are as per the following in [2015]:

Distributed actions: No other backdrop system for the essential reign of the system action. The reign of the system is disseminated amid the hops. The hops that perform in a MANET would coordinate with one another and transmit among themselves and every hub capacities as a hand-off as required, to satisfy explicit capacities, for example, routing and security.

Multi-hop Routing: In the event that a hop needs to transfer data to other hop which is exterior of its correspondence space, the packet needs to retransfer through at least one moderate hop.

Autonomous terminal: In MANET, each portable hop is a self-reliant hop. It can accomplish two errands they can work as host and router.

Dynamic topology: In mobile Ad hoc network the system structure may constantly adjust and at eccentric time. The hops are allowed to proceed self-assertively in the system domain with

Different speeds. Along these lines, the hops in the mobile Ad hoc network progressively set up routing in the middle themselves as they carrying around shaping their very own system.

Hared Physical Medium: The wireless transmission medium is portrayed as available to any substance with the correct hardware and sufficient assets. Respectively, entrance to the channel can't be restricted.

\subsection{APPLICATIONS OF MANETS}

The MANET is these days so promoted that it has numerous supplications in a few area in the real life. The following are the fundamental supplications for MANET [2]:

Emergency services: MANET is utilized in numerous activities at crisis time. For instance, it is utilized in explore and recovery actions. At the point when emergency happens, MANET ends up important in that mishap revelation and recovery. It is likewise helpful on account of ecological crises, for it substitute the wired structure. It is valuable in medicinal region, since it backing doctors and assistants in clinics.
Tactical Networks: MANET has much utilization in the army. A portion of this utilization is in the army divulgence and actions among armed force members. Also, it is utilized in the computerized combat zone.

Home and enterprises networking: MANET works as home/office wireless systems. It is utilized for swap information in the seminar and gathering spaces. It is utilized personal area networks (PAN) and personal network (PN). It is also utilized as systems at building locales.

Educations: MANET is of extensive significance in the academic sections. It is unavoidable in the colleges and branch setting. It is crucially required in virtual study halls. Also, it is utilized in Ad hoc transmissions throughout the sessions or lessons. Sensor network: MANET is valuable in pursue on favor like call sending and portable escritoire. Also, it is utilized in data utility e.g. area explicit administrations, time subordinate services. It is utilized in the touristic segment for transmission data.

\section{$\begin{array}{llll}1.4 & \text { CLASSIFICATION } & \text { OF } & \text { ROUTING }\end{array}$} PROTOCOLS MANET routing protocol need to adjust quick to the regular changes in topology that is likewise unreliable, it must be successful in appropriately using the system resources. The protocols are classified into three: proactive, reactive and hybrid [2015]. Figure-4 shows the classification of MANET routing protocols.

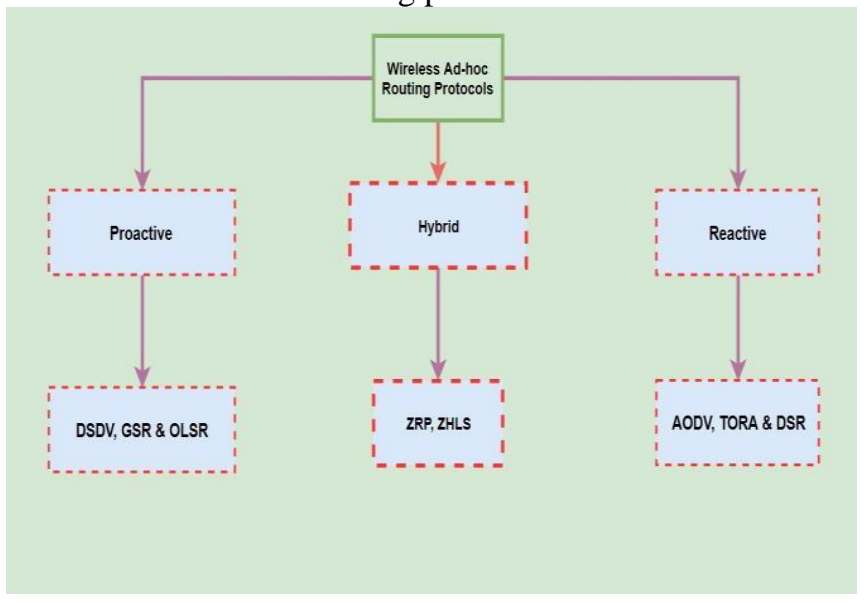

Figure 4. Classification of routing protocols

\section{a) $\mathbf{R e}$-active protocols}

Re-active routing protocol gives a route to on interest flow of data. Subsequently, routes are chosen and kept up for hops that need to send information to a particular destination. However, the hub could stay inactive or dynamic for taking an interest during the time spent sending for serving other source hops. Re-active protocols devour fewer assets while the hops stay inert for finding a route (as they don't have any data with respect to topology) [2015].

Dynamic Source Routing (DSR): Dynamic Source Routing is a Pure on-Demand routing protocol, where the route is determined just when it is fundamental. It is intended for use in multi-hop Ad hoc networks of portable hops. DSR enables the system to be self-sorted out and self-designed without main devices. It utilizes no repeated routing messages like AODV, along these lines decreases data transmission 


\section{International Journal of Engineering Applied Sciences and Technology, 2019 \\ Vol. 4, Issue 8, ISSN No. 2455-2143, Pages 356-361 \\ Published Online December 2019 in IJEAST (http://www.ijeast.com)}

overhead and saved battery control and furthermore tremendous routing updates. It needs just the intention from the MAC layer to distinguish interface disappointment's uses source routing where the entire route is conveyed as an overhead.

\section{b) Pro-active protocols}

The routing procedure in table driven protocols relies upon pre-defined routing tables which comprise of routes to all receivers on the system. These routing tables are occasionally refreshed to include each refurbish that can appear to the system. All through the system, table driven protocols are intermittently disseminating the routing tables. Notwithstanding, and particularly in the broad networks table driven protocols can matter additional upward as they are enforced to accomplish updated routing data to fulfill the best possible routing procedure, and accordingly decrease the system throughput [2015].

Destination-Sequenced Distance-Vector (DSDV): DSDV is one of the table-driven or proactive routing protocols based on the Bellman-Ford Algorithm, created by C. Perkins and P. Bhagwat in 1994. One of the crucial commitments of the Algorithm was to appliance the routing loop problem. The routing table comprises of different passages, and each entry comprises of a sequence number that is regardless of whether a connection is available else, it is odd. Routing data is disseminated between hops by sending full dumps once in a while and smaller incremental updates all the more as often as possible [2015].

\section{c) Hybrid routing protocols}

These protocols combine the great equity of the proactive and re-active routing protocols. It aggregates the bundle of hops into area in the system topology. As the system is part into areas, the proactive methodology is utilized inside each area for support of routing data. Re-active methodology is utilized for routing packets between various zones.

Zone routing protocol (ZRP): Zone Routing Protocol is a hybrid routing protocols that isolates the system into zones. ZRP gives a progressive architecture where every hub needs to keep up extra topological data requiring additional memory.

\section{LITERATURE REVIEW}

The work of OLSR, DSR and AODV using OPNET 14.5 and NS-2 Network simulator has been investigated in [2014]. Performance matrices were used are average packet transmission delay, throughput, end-to-end delay. The simulation Outcome shows that OLSR performs well in term of throughput as compare to DSR and AODV. In term of retransmission attempt AODV has great load and in term of media access delay DSR performs well as compare to OLSR and AODV. The work of AODV, DSDV and DSR using OMNET++, NS-2 and GloMosim network simulators has been investigated in [2014]. Performance matrices were used are throughput, the packet delivery ratio and the energy consumption. The first experiment has been done by NS-2 for AODV, DSDV and DSR their results shows that DSR performs well under varying pause time, energy consumption, node mobility, random mobility. Other experiment done for AODV and DSDV the result shows that DSDV has dominate fewer energy as compare to AODV. The effort of AODV, DSR, DYMO, and LAR using Qualnet 5.2 network simulator has been investigated in [2014]. Performance matrices were used are throughput, packet delivery ratio, jitter, end to end delay, total energy consumption under mobility and energy model. Their result shows that LAR performs well in term of average jitter and end to end delay in delicate applications as compare to all matrices and in mobility models. AODV performs well in term of PDR when number of nodes going to high in Random Mobility model and DSR performs well for PDR in Group Mobility model. DSR better as compare to other protocols in term of throughput when number of nodes increases The effort of AODV, DSR and DSDV using NS -

\subsection{5 network simulator has been investigated in}

[2017]. Performance matrices were used are PDR, throughput, end-to-end delay, and PLR. There simulation outcome shows that DSDV performs well as compare to DSR and AODV in term of PDR and PL, while AODV performs well in term of TP as compare to DSDV and DSR. DSR perform well than AODV and DSR in term of end

$$
\text { - to - end delay. }
$$

The work of Dynamic Source Routing (DSR), Destination Sequenced Distance Vector (DSDV), On-demand Distance Vector (AODV), Zone Routing Protocol (ZRP), and Temporally Ordered Routing Algorithm (TORA) using NS 2.35 has been investigated in [2018]. Performance matrices were used are sent packets, received packets, PDR, throughput, end to end delay, packets dropped, sending jitter and receiving jitter are analyzed. There outcome shows that the performance of all protocols turn to the number of hops. The effort of DSR, DSDV and AODV using NS -

2.35 network simulator has been investigated in [2018]. Performance matrices for analysis were used are throughput, PDR and end-to-end delay. AODV performs well than DSR and DSDV in high density networks, while DSR performs better than AODV in low density network than DSR and DSDV.

\section{SIMULATIONS SETUP}

NS-2 Simulation is utilized by various analysts because of it is extraordinary preferred advantage over different simulators for example, calculation time it lessens the CPU use when other application are executed in parallel. In this Segment we have communicated the performance perception of MANET's routing protocols. The propagation time is taken as $100 \& 50$ seconds. Table -1 demonstrates the networks and characteristics utilized for analyses. 


\section{International Journal of Engineering Applied Sciences and Technology, 2019 \\ Vol. 4, Issue 8, ISSN No. 2455-2143, Pages 356-361 \\ Published Online December 2019 in IJEAST (http://www.ijeast.com)}

Table 1. Simulation parameters

\begin{tabular}{|l|l|l|}
\hline S.No & Parameters & Values \\
\hline 1 & Area & $\begin{array}{l}400 \times 400,600 \times 600, \\
800 \times 800\end{array}$ \\
\hline 2 & Hops assignments & Random \\
\hline 3 & Source & CBR \\
\hline 4 & No of hops & $10,25 \& 50$ \\
\hline 5 & Simulation time & $50 \& 100$ sec \\
\hline 6 & Protocols & DSR, DSDV \& ZRP \\
\hline 7 & Operating System & Ubuntu 16.04 \\
\hline 8 & MAC protocols & 802.11 \\
\hline
\end{tabular}

a) Throughput: Network throughput makes reference to the normal data rate of effective information or message conveyance over a particular correspondences link.

b) Normalized Routing Load: NRL is introducing as the overall figure of routing packet transferred per data packet.

c) End to end delay: End -to - end delay is the time it traps to send the whole message from sender to receiver.

d) Packet delivery ratio: $\mathrm{PDR}$ is the rate of packets well acknowledged to the overall sent.

\section{RESULTS AND DISCUSSIONS}

In this analysis we have done the experiments with NS 2.35 network tool. We compared three main protocols type reactive, proactive and hybrid routing protocols by varying performance Parameters like Normalized routing overhead, end

- to - delay, throughput and packet delivery ratio in various pause time and number of hops.

From Figure 5, different routing protocols PDR has been analyzed through simulation, it shows that DSDV has started with lower value and end with zero while increases in middle number of hops. DSR started with high value and decreases from low to high number of hops. ZRP is started with lower value but increases from lower to high number of hops.

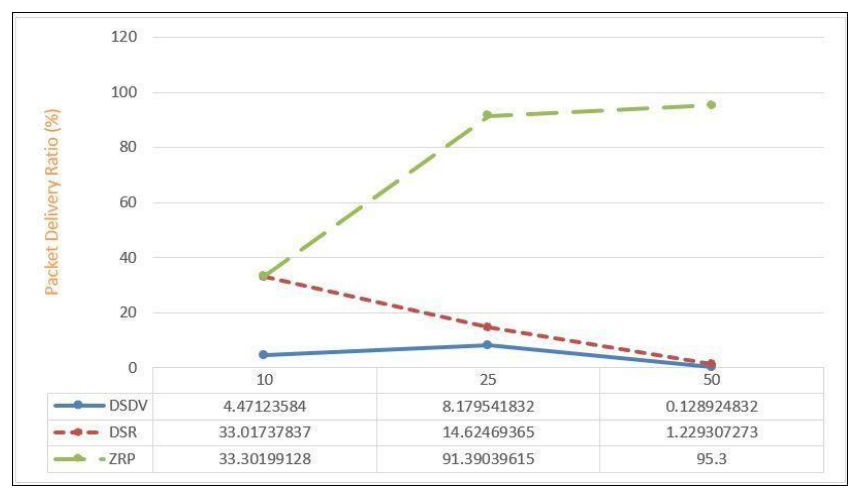

Figure 5- Packet delivery Ratio Vs. No of Nodes

From Figure $6 \& 7$, it shows that DSDV \& DSR starts with moderate value and decreases from lower to higher number of hops, but ZRP starts with lower value and increases from lower to high number of hops.

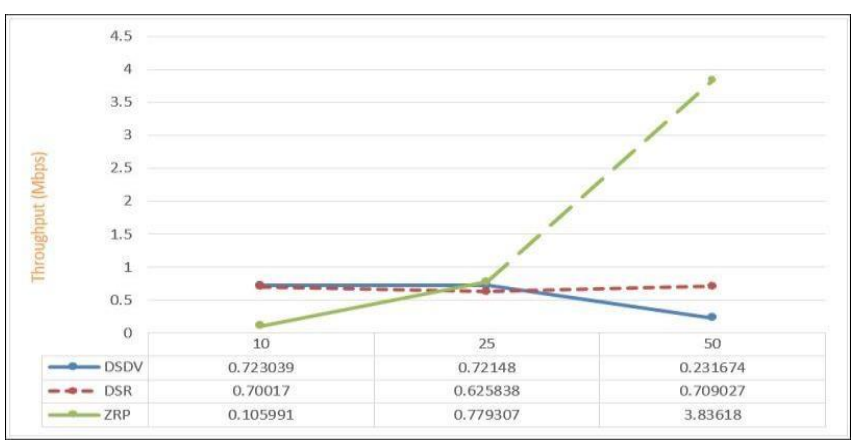

Figure 6. Throughput vs. Number of Nodes 100 pause time

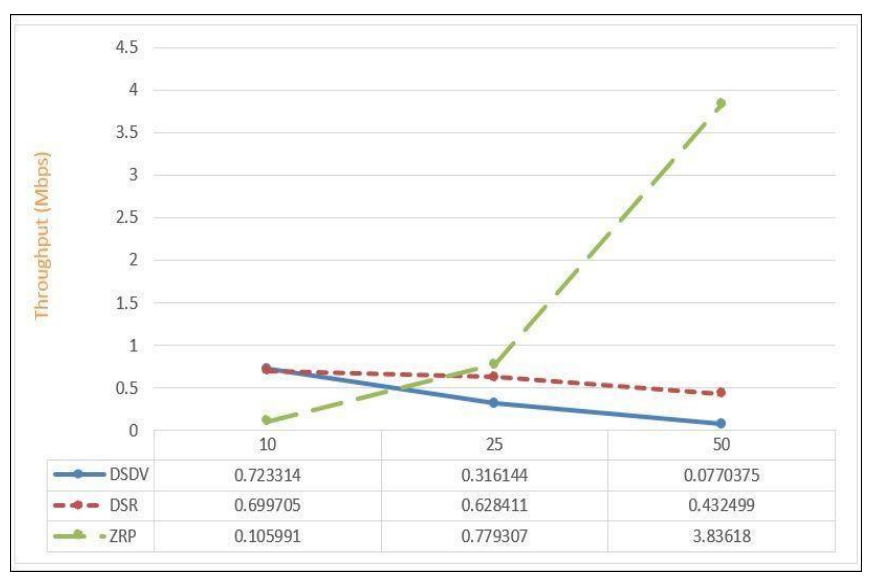

Figure 7. Throughput vs. Number of Nodes 50 pause time 


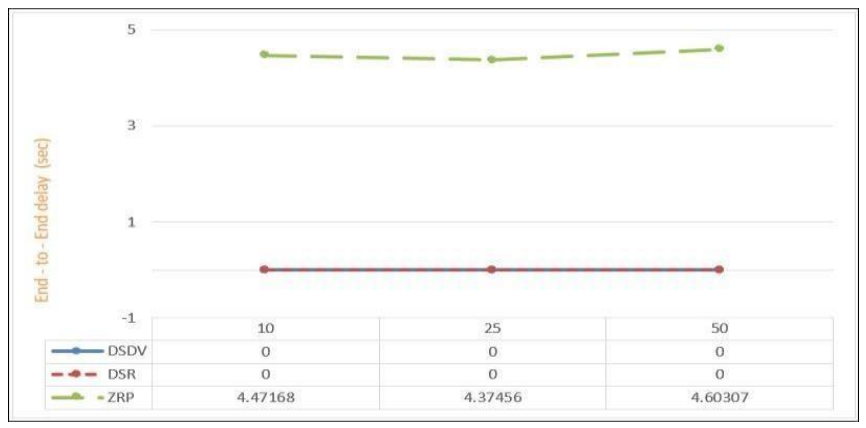

Figure 8. E2E delay vs. Number of Nodes 100 pause time

From Figure $8 \& 9$, it is shows that DSDV and DSR end - to - end delay is zero always while ZRP starts with a higher value and remain same.

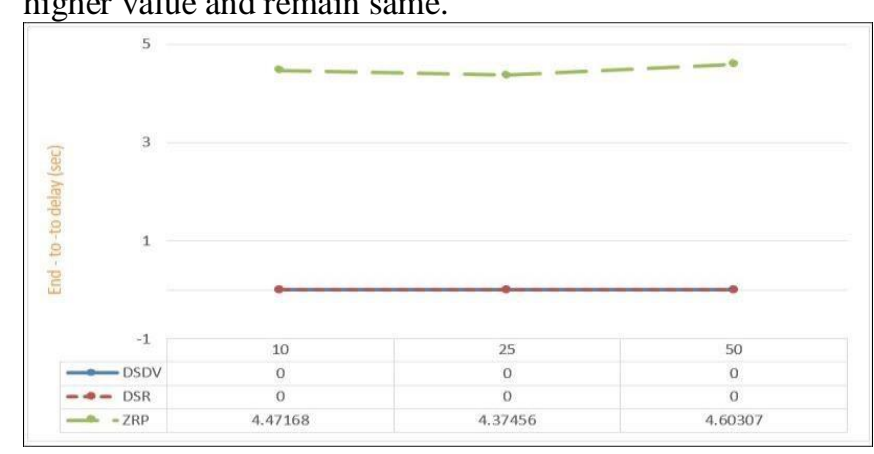

Figure 9. E2E delay vs. Number of Nodes 50 pause time

From Figure 10, it shows that DSDV and ZRP NRL value starts with lower and increases from lower to

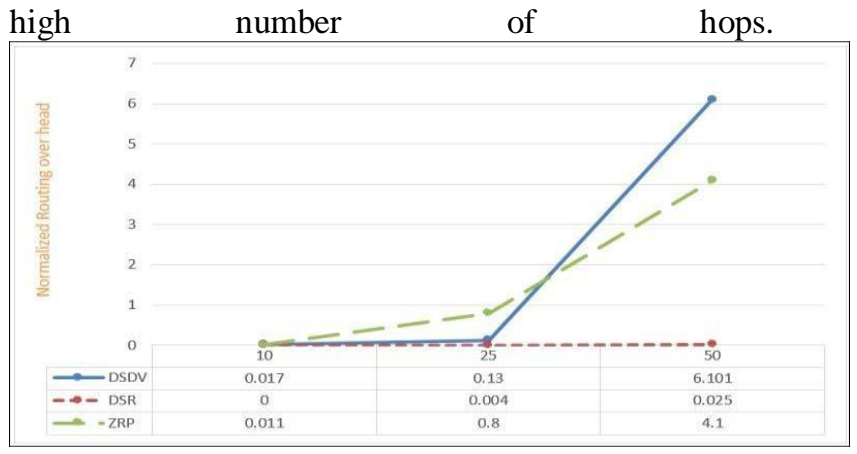

Figure 10. NRL vs. Number of Nodes 100 pause time

From Figure 11, it shows that DSDV \& DSR NRL value starts with lower but increases from lower to high number of hops. ZRP starts from moderate value and increases to higher.

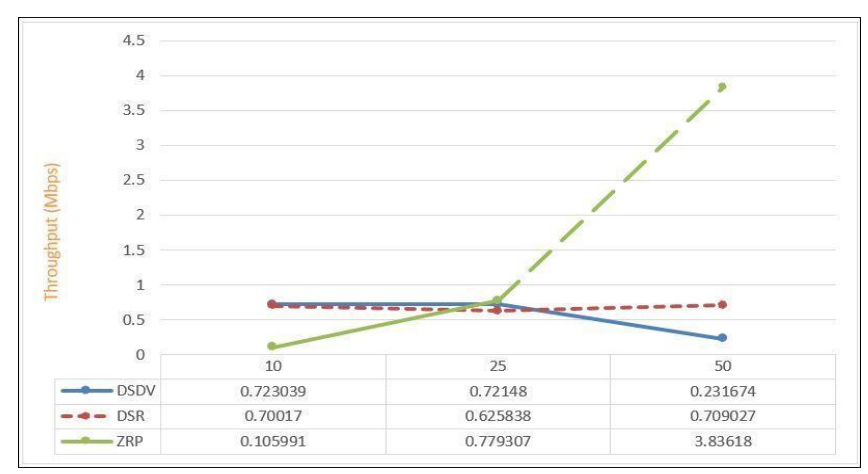

Figure 11. NRL vs. Number of Nodes 50 pause time

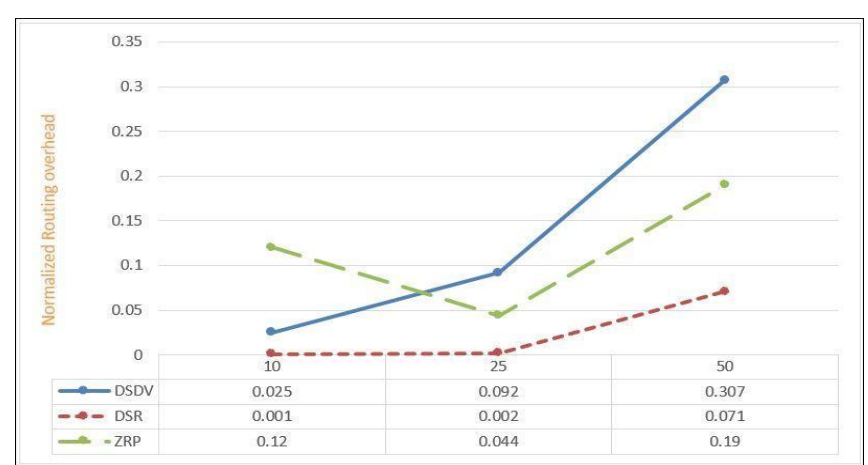

Figure 12. Throughput vs. Number of Nodes 50 pause time

From Figure 12, it shows that DSDV \& DSR starts with moderate value and decreases in high number of hops while ZRP starts with lower value but increases with high number of hops.

\section{CONCLUSION}

Routing is the crucial part in networking to transfer data packets by efficient way. This research study work is to analyze and compare the proactive, reactive and hybrid routing protocols for performance matrices normalized routing overhead, throughput, end - to end delay and packet deliver ratio in varying number of hops and simulation time. Our result shows that ZRP will be better to use in those scenarios where number of hops are increases and best packet delivery ratio and throughput is crucial requirements while DSDV and DSR will be better to use where excellent end - to - to delay is required.

\section{REFERENCES}

[1] Jatinder Kaur Er., \&. Gurpreet Singh Er, (2017)"Review study on MANET Routing Protocols: Challenges and Applications," International Journal of Advanced Research in Computer Science, pp. 140 - 145.

[2] Ali Abdalftah Kaid Said., Kulkarni Dr. U. V (2015). "Characteristics, Applications and Challenges in Mobile AdHoc Networks (MANET): Overview," IPASJ International Journal of Electronics \& Communication (IIJEC), vol. 3, no. 
12, pp. $6-12$,

[3] Raaid Alubady., Al-Samman Mohammad., Habbal Adib., Hassan Suhaidi., Arif Suki., (2015) "PERFORMANCE ANALYSIS OF REACTIVE AND PROACTIVE ROUTING PROTOCOLS IN MANET," ARPN Journal of Engineering and Applied Sciences, vol. 10, no. 3 , pp. $1468-1478$.

[4] A. Elsadig Muawia,. A. Fadlalla Yahia (2018) "Mobile Ad Hoc Network Routing Protocols: Performance Evaluation and Assessment," International Journal of Computing and Digital Systems, vol. 7, no. 1, pp. 59 - 66, Jan-2018.

[5] Mummadisetty Bharath Chandra., Puri Astha. Latifi Shahram (2015) "Performance Assessment of MANET Routing Protocols," Int. J. Communications, Network and System Sciences, vol. 8, pp. 456-470.

[6] Atif Mahmood., Buttar Mr. Ahmad Mateen., Irfan Ali., M. Arslan Saeed Khan (2014) "Performance Analysis of Proactive OLSR and Reactive DSR, AODV protocols for MANET," International Journal of Innovation and Scientific Research, vol. 10 , no. 2, pp. 498-506.

[7] Shastri Dipti., Lala Ajay (2017) "Comparison of AODV, DSR and DSDV on Different Simulators for QoS Parameters," International Journal of Science and Research (IJSR), vol. 6, no. 1, pp. $1029-1033$.

[8] Goyal Er. Hanisha., Kakkar Er. Parveen., (2014) "Performance Investigation of DYMO, DSR, AODV and LAR Routing Protocols using Different Mobility and Energy Models in MANETs," International Journal Of Engineering And Computer Science, vol. 3, no. 3, pp. 50175027.

[9] AL-Dhief Fahad Taha., Naseer Sabri., Salim M.S., Fouad S., Aljunid S. A. (2017) "MANET Routing Protocols Evaluation: AODV, DSR and DSDV Perspective," Malaysia, MUCET.

[10] Ahmed Sheeraz., Bilailr Muhammad., Farooq Umer., Fazl-e-Hadi (2018) "Performance Analysis of various Routing Protocols in Mobile Ad-hoc Networks," International Journal of Applied Engineering Research, vol. 13, no. 10, pp. 73787382. 